\title{
On the Existence of Fixed Points of the Composition Operator for Circle Maps
}

\author{
J.-P. Eckmann ${ }^{1}$ and H. Epstein ${ }^{2}$ \\ ${ }^{1}$ Département de Physique Théorique, Université de Genève, Bd. d'Yvoy, CH-1211 Genève, \\ Switzerland \\ ${ }^{2}$ Institut des Hautes Etudes Scientifiques, 35 route de Chartres, F-91440 Bures sur Yvette, France
}

\begin{abstract}
In the theory of circle maps with golden ratio rotation number formulated by Feigenbaum, Kadanoff, and Shenker [FKS], and by Ostlund, Rand, Sethna, and Siggia [ORSS], a central role is played by fixed points of a certain composition operator in map space. We define a common setting for the problem of proving the existence of these fixed points and of those occurring in the theory of maps of the interval. We give a proof of the existence of the fixed points for a wide range of the parameters on which they depend.
\end{abstract}

\section{Introduction}

Fixed points for composition operators are now known to exist in a multitude of situations: maps of the interval, dissipative maps of $\mathbb{R}^{n}$, area preserving maps of the plane and circle maps all possess fixed points. In this paper, we try to connect the cases of interval maps and circle maps (with golden rotation number) by giving an interpolation between the two. In fact, there is a two parameter family of problems, namely to find solutions of the equations

with

$$
\phi(x)=-\frac{1}{\lambda} \phi\left(\frac{1}{\lambda^{v-1}} \phi\left(\lambda^{v} x\right)\right)
$$

$$
\phi(x)=f\left(x^{r}\right), \quad \phi\left(\frac{1}{\lambda^{v-1}}\right)=-\lambda, \quad \phi(0)=1, \quad \lambda \in(0,1),
$$

and $f$ analytic on $[0,1]$. Here, the two parameters are $r$ and $v$. The case $v=1$ corresponds to maps of the interval, while the case $v=2$ corresponds to circle maps. The value $r=2$ is of main interest for the physics corresponding to the case $v=1$, and $r=3$ is of interest when $v=2$. For the possible occurrence of different $r$ in dynamical systems, see [ACT]. [A more precise statement of the problem is embodied in Eqs. (1.19), (1.20) below.]

Consider the region $D$ given by

$$
D=\left\{(\lambda, v) \in \mathbb{R}^{2}: 1 \leqq v \leqq 2,0<\lambda<1, \lambda^{v}+\lambda^{v-1}-1>0\right\} .
$$




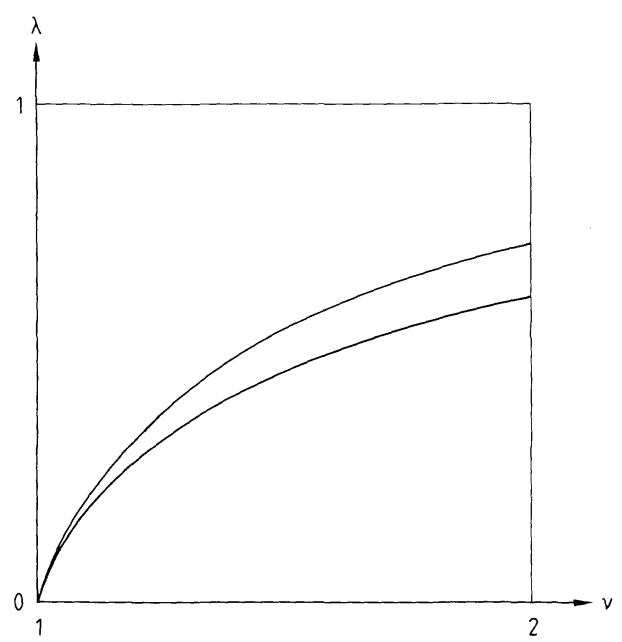

Fig. 1. The domain $D$ is above the lower curve. The obtained domain is above the upper curve

We conjecture that for every $(\lambda, v) \in D$, Eq. (1.1) has a solution which is analytic in $\lambda$ and $v$ in the interior of $D$. Our results go some way towards proving this conjecture, but fail to exhaust $D$ and to provide the conjectured analyticity. Figure 1 shows the expected and the obtained domains of existence of $\phi$.

The proof given here extends that in [E]. The main observation (see [EL]) is that the inverse function of the function $f$ has the (anti-)Herglotz property, i.e., $-f^{-1} \equiv-U$ maps the upper half plane into itself. Therefore it has an integral representation with positive weight, from which simple, but useful inequalities can be derived. These then serve to prove the existence of solutions by an application of the Schauder-Tikhonov theorem. For technical reasons, we are forced to fix $\lambda$ and $v$ in our construction, instead of the more desirable $r$ and $v$, and because we use the Schauder-Tikhonov theorem, we are not able to obtain the continuity of $r(\lambda, v)$.

\subsection{Notations}

Let $J$ be an open, possibly empty interval in $\mathbb{R}$. We denote

$$
\mathbb{C}(J)=\{z \in \mathbb{C}: \operatorname{Im} z \neq 0 \text { or } z \in J\}
$$

In particular, $\mathbb{C}(\varnothing)=\mathbb{C}_{+} \cup \mathbb{C}_{-}$, where

$$
\mathbb{C}_{+}=-\mathbb{C}_{-}=\{z \in \mathbb{C}: \operatorname{Im} z>0\} .
$$

$\mathbf{F}(J)$ is the real Fréchet space of functions $f$, holomorphic on $\mathbb{C}(J)$, with $f\left(z^{*}\right)^{*}$ $=f(z)$, equipped with the topology of uniform convergence on compact subsets of $\mathbb{C}(J)$.

$\mathbf{P}(J)$ is the subset of $\mathbf{F}(J)$ consisting of the functions $f$ such that $f\left(\mathbb{C}_{+}\right) \subset \overline{\mathbb{C}}_{+}$, and $f\left(\mathbb{C}_{-}\right) \subset \overline{\mathbb{C}}_{-}$. These functions are often called Herglotz or Pick functions.

$\mathbf{P}_{0}(J)$ is the subset of $\mathbf{P}(J)$ consisting of the functions $f$ such that $|f(z) / z| \rightarrow 0$ as $z \rightarrow \infty$ in non-real directions. 
Note that if $h$ belongs to $\mathbf{P}(J)$ and is not constant and real in $\mathbb{C}_{+} \cup \mathbb{C}_{-}$, then $h\left(\mathbb{C}_{+}\right) \subset \mathbb{C}_{+}$. If, moreover, $J$ is not empty, then on $J, h^{\prime}$ is strictly positive, and $S h=h^{\prime \prime \prime} / h^{\prime}-(3 / 2)\left(h^{\prime \prime} / h^{\prime}\right)^{2}$, the Schwarzian derivative of $h$, is positive. Recall that, if a smooth function $h$ has a non-vanishing derivative and $S h \geqq 0$, then its inverse function has negative Schwarzian and conversely.

In this paper the function $\log$ is defined in $\mathbb{C}((0,+\infty))$ so that it is real on $\mathbb{R}_{+}$, and $z^{s}=\exp (s \log z)$.

\subsection{The Circle Case}

We now formulate the problem for the case $v=2$, i.e. for circle maps with golden rotation number [FKS, ORSS, JR, N, LL, M, L1, L2]. The natural $v$-dependence will reappear in Eq. (1.19). We follow the exposition of Lanford [L1, L2] and use the notations of [L2]. We consider the problem of finding solutions $(\xi, \eta)$ of the equations:

$$
\begin{gathered}
\eta(x)=-\frac{1}{\lambda} \eta(\xi(-\lambda x)), \\
\xi(x)=-\frac{1}{\lambda} \eta(-\lambda x), \\
\xi(0)=1
\end{gathered}
$$

which satisfy the constraints $\mathrm{C} 1-\mathrm{C} 3$ discussed below. [In this case the function $\phi$ is given by $\phi(x)=\xi(-x)$.]

C1. $\eta$ is a strictly increasing continuous function on an interval $[0, L]$, where it vanishes at a certain $x_{0} \in(0,1)$, and $\eta(0)=-\lambda$, with $\lambda \in(0,1)$. The function $\xi$ defined by (1.3) has corresponding properties. For all $x$ in $[0, L], \xi(-\lambda x)$ is also in $[0, L]$ and (1.2) holds. In particular $1 \leqq L x_{0} \lambda^{-2}$.

C2. There exists a real $r>1$, and a function $G$, holomorphic without critical points on a complex neighborhood of $\left[0, L^{r}\right]$, (in particular $G^{\prime}(0) \neq 0$ ), such that, on $[0, L]$,

$$
\eta(x)=G\left(x^{r}\right) \text {. }
$$

Correspondingly, there exists a function $F$, holomorphic on a complex neighborhood of $\left[-(L / \lambda)^{r}, 0\right]$, such that, for all $x \in[-L / \lambda, 0]$,

$$
\xi(x)=F\left(-(-x)^{r}\right),
$$

and, for all $t \in\left[-(L / \lambda)^{r}, 0\right]$,

$$
F(t)=-\frac{1}{\lambda} G(-\tau t), \quad \tau=\lambda^{r} .
$$

The consequences of $\mathrm{C} 1$ and $\mathrm{C} 2$ are discussed in the papers of Nauenberg and Lanford [N, L1, L2]. Here we need the following facts:

1. The unique solution $x_{0}^{\prime}$ in $\left(0, x_{0} / \lambda^{2}\right)$ of the equation $\xi\left(-\lambda x_{0}^{\prime}\right)=x_{0}^{\prime}$ satisfies $x_{0}^{\prime} \in(0,1)$ and, by (1.2), $\eta\left(x_{0}^{\prime}\right)=0$, hence it coincides with $x_{0}$ and

$$
\xi\left(-\lambda x_{0}\right)=x_{0}
$$


2. By using (1.2-1.4), $\eta$ can be extended to a strictly increasing function on $\left[0, x_{0} / \lambda^{2}\right]$ and

$$
\eta\left(x_{0} / \lambda^{2}\right)=1, \quad \eta^{\prime}\left(x_{0} / \lambda^{2}\right)=0
$$

Near this point,

$$
\eta(x) \sim 1-\operatorname{const}\left(x_{0} / \lambda^{2}-x\right)^{r}
$$

3. Note that

$$
\begin{gathered}
-\lambda=\eta(0)=-\frac{1}{\lambda} \eta(\xi(0)), \quad \text { i.e. } \quad \eta(1)=\lambda^{2}, \\
\eta\left(x_{0} / \lambda^{2}\right)=1>\lambda^{2}, \text { hence } x_{0} / \lambda^{2}>1 .
\end{gathered}
$$

4. Since, for some $\varepsilon>0, G(t)$ is analytic and real in $\left(-\varepsilon, L^{r}\right]$, the functional equation

$$
G(t)=-\frac{1}{\lambda} \eta\left(-\frac{1}{\lambda} G\left(\tau^{2} t\right)\right)
$$

an immediate consequence of (1.2), (1.3), and (1.5) for $t \in\left[0,\left(x_{0} / \lambda^{2}\right)^{r}\right]$, can be used to continue $G$ (respectively $F$ ) on the negative (respectively positive) real axis as a strictly increasing, analytic function, with $G(t)<-\lambda$ for $t<0$. There are two possibilities:

a) for some $t=-\tau^{2} t_{2}<0$, (the extension of) $G(t)$ takes the value $-x_{0} / \lambda$. Then $G$ can be extended to $\left[-t_{2}, 0\right]$, with $G\left(-t_{2}\right)=-1 / \lambda$. Correspondingly, $F$ can be extended as a strictly increasing, continuous function on $\left[-\left(x_{0} / \lambda^{3}\right)^{r}, t_{1}\right]$, with $t_{1}>0$, and

$$
F\left(-\left(x_{0} / \lambda^{3}\right)^{r}\right)=-\frac{1}{\lambda}, \quad F\left(t_{1}\right)=\frac{1}{\lambda^{2}} .
$$

$F$ is analytic inside this interval and has critical points at its ends.

b) The extension of $G$ never reaches the value $-x_{0} / \lambda$. In this case the righthand side of (1.11) is always defined and can be used to define $G(t)$ for all $t<0$. As $t \rightarrow-\infty, G(t)$ decreases and tends to a limit $G(-\infty) \in\left[-x_{0} / \lambda, 0\right)$, which must be a fixed point of $-\lambda^{-1} \eta \circ\left(-\lambda^{-1}\right)$. Thus case $\left.b\right)$ is ruled out by the condition that this function have no other fixed point, in its interval of definition $\left[-x_{0} / \lambda, 0\right]$, than $-\lambda$ (see (1.10)), or, equivalently by the condition

P1. The function $\Phi \equiv \lambda^{-2} \eta$ has no other fixed point than 1 in $\left[0, x_{0} \lambda^{-2}\right]$.

Case b) will be ruled out by our condition C3 below. It is interesting, however, to note that the condition $\mathbf{P 1}$ is implied by the weaker assumption that $\eta$, and hence $\Phi$ have negative Schwarzian. If so, denoting $w=\Phi^{-1}$, then $w$ has positive Schwarzian, i.e. on $\left(-\lambda^{-1}, \lambda^{-2}\right), w$ is analytic, strictly increasing, and

$$
S w \equiv \frac{w^{\prime \prime \prime}}{w^{\prime}}-\frac{3}{2}\left(\frac{w^{\prime \prime}}{w^{\prime}}\right)^{2} \geqq 0, \text { hence }\left(\frac{w^{\prime \prime}}{w^{\prime}}\right)^{\prime} \geqq 0 \text {. }
$$

Moreover $w$ is continuous on $\left[-\lambda^{-1}, \lambda^{-2}\right]$, with $w\left(-\lambda^{-1}\right)=0, w\left(\lambda^{-2}\right)=x_{0} \lambda^{-2}$, $w^{\prime}\left(-\lambda^{-1}\right)=w^{\prime}\left(\lambda^{-2}\right)=+\infty$, and $w(0)=x_{0}$. Since $x_{0} \lambda^{-2}<\lambda^{-2}$, the fixed points 


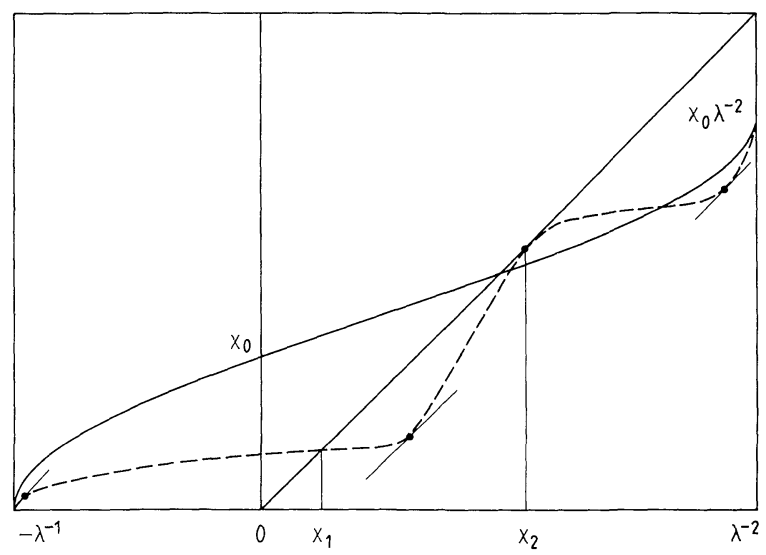

Fig. 2. Solid line: graph of $w$ as it must be. Dotted line: impossible situation

must be in $\left(0, \lambda^{-2}\right)$. If $x_{1}<x_{2}$ are two distinct fixed points, $w^{\prime}$ must take the value 1 in $\left[x_{1}, x_{2}\right]$. Moreover, $w^{\prime}$ must take values $<1$ in $\left(-\lambda^{-1}, x_{1}\right)$ (since otherwise $w(z)$ $\geqq z+\lambda^{-1}$ in this interval), and because $w^{\prime}\left(-\lambda^{-1}\right)=+\infty, w^{\prime}$ must go through 1 there. For the same reasons it must also take the value 1 in $\left(x_{2}, 1\right)$. But $\log w^{\prime}$ is convex by (1.12), and can vanish at most twice in $\left(-\lambda^{-1}, \lambda^{-2}\right)$. Hence there is exactly one fixed point of $w$ in $\left[-\lambda^{-1}, \lambda^{-2}\right]$ (see Fig. 2).

C3. We require the inverse function $-U$ of $F$ to be a Herglotz function:

$$
U \equiv-\left(F^{-1}\right) \in-\mathbf{P}\left(\left(-\lambda^{-1}, \lambda^{-2}\right)\right) .
$$

Since the identity $\xi(x)=F\left(-(-x)^{r}\right)$ extends to $\left(-x_{0} / \lambda^{3}, 0\right)$, denoting $-u$ the inverse function of $\xi$ restricted to this interval (i.e. $u$ is the inverse function of $\phi$ ), we get:

$$
u(z)=[U(z)]^{1 / r} .
$$

This holds in $(-1 / \lambda, 1)$, and extends by analytic continuation to $\mathbb{C}_{+} \cup \mathbb{C}_{-}$, so that

$$
u \in-\mathbf{P}\left(\left(-\lambda^{-1}, 1\right)\right)
$$

Comment on $C 3$. The requirement $\mathbf{C 3}$ is imposed by analogy with the case of maps of the interval. A further justification is that it is implied by the other (hyperbolicity) conjectures usually made [FKS, ORSS, N, L1, L2] and, in certain cases, proved [JR, M], about the solutions of (1.2), (1.3) (and which, in fact, make these solutions interesting for dynamical systems theory). If the standard picture holds then the "shift operator" $T$,

$$
T\left(\begin{array}{l}
\xi \\
\eta
\end{array}\right)(x)=\left(\begin{array}{c}
-\frac{1}{\lambda} \eta(-\lambda x) \\
-\frac{1}{\lambda} \eta(\xi(-\lambda x))
\end{array}\right), \quad \lambda=-\eta(0),
$$

has a one-dimensional unstable manifold going through the fixed point (at fixed $r$ ). Under repeated action of $T$, this manifold attracts neighboring curves [i.e. one- 
parameter families of pairs $(\xi, \eta)]$ in function space. Among such families, many will consist of pairs whose inverse functions have the Herglotz property as implied by C3. This property is stable under $T$. Vitali's theorem then implies that the convergence of these functions on the reals also holds in the complex cut plane. Hence, at least near the fixed point, all the maps forming the unstable manifold have Herglotz inverse functions. This holds true along the unstable manifold as far as it can be extended by repeated application of $T$. The same argument shows that these inverse functions have univalent restrictions to $\mathbb{C}_{ \pm}$, since this property is also stable under $T$, and stable under limits by Hurwitz's theorem. In the present state of our method, we are not able to use (or prove) this univalence because it does not define a convex set in $\mathbf{P}(I)$.

To take advantage of $\mathbf{C} \mathbf{3}$, we introduce the following notations:

$$
\begin{gathered}
\psi(z)=\frac{\tau}{y_{0}} U(z), \quad \tau=\lambda^{r}, \quad y_{0}=x_{0}^{r}, \\
V(\zeta)=\frac{1}{\tau^{2}} \psi\left(x_{0} \zeta^{1 / r}\right) .
\end{gathered}
$$

The functional equations (1.2-1.7) then imply:

$$
\begin{gathered}
\psi(z)=V(\psi(-\lambda z)), \\
\psi(0)=1, \quad \psi(1)=0, \quad V(1)=1, \quad V^{\prime}(1)=-\frac{1}{\lambda} .
\end{gathered}
$$

Conversely suppose that two functions $\psi$ and $V$ satisfy

$$
\begin{gathered}
\psi \in-\mathbf{P}\left(\left(-\lambda^{-1}, \lambda^{-2}\right)\right), \quad V \in-\mathbf{P}\left(\left(0, \alpha / \tau^{2}\right)\right), \\
\psi(0)=1, \quad \psi(1)=0, \quad V(1)=1, \quad V^{\prime}(1)=-\frac{1}{\lambda}, \\
\psi(z)=V(\psi(-\lambda z)), \\
V(\zeta)=\frac{1}{\tau^{v}} \psi\left((\zeta / \alpha)^{1 / r}\right), \\
0<\lambda<1, \quad \tau=\lambda^{r}, \quad r>1, \quad 1<\alpha<\tau^{-v},
\end{gathered}
$$

with: $v=2$. Then setting $x_{0}=\alpha^{-1 / r}$, we obtain a solution of the original equations satisfying C1-C3. We note that the same equations, but with $v=1$, are equivalent to the functional equation occurring in the case of maps of the interval. In Eq. (1.19), the interpolating parameter $v$ has reappeared. Given a solution of (1.19), (1.20), one obtains a solution of (1.1) as follows: the inverse function of $\phi$ is given by

$$
\phi^{-1}(z) \equiv u(z)=\frac{1}{\lambda^{v-1}}\left[\alpha^{-1} \psi(z)\right]^{1 / r} .
$$

\section{The Question of Commutativity}

The theory leading to Eqs. (1.2-1.7) also requires that $\xi$ and $\eta$ commute. References $[\mathrm{N}, \mathrm{L} 1]$ give this property a precise meaning and show that the property $\mathbf{P 1}$ stated 
in Sect. 1 is a necessary and sufficient condition for it. In this section we transcribe their arguments in our notation to show how C1-C3 directly imply commutativity. Starting from

$$
\begin{gathered}
u=\frac{1}{\lambda^{2}} u \circ \lambda \circ u \circ(-\lambda), \\
U=\frac{1}{\tau^{2}} U \circ \lambda \circ u \circ(-\lambda),
\end{gathered}
$$

we define

$$
\tilde{U}=-\frac{1}{\tau} U \circ \frac{1}{\lambda} \circ u \circ \lambda^{2}
$$

Note that (2.2) and (2.3) are identities that hold together with domains, namely (2.2) holds in $\mathbf{C}\left(\left(-\lambda^{-1}, 1\right)\right)$, and (2.3) holds in $\mathbf{C}\left(\left(-\lambda^{-1}, \lambda^{-2}\right)\right)$. It is easy to verify that $\tilde{U} \in-\mathbf{P}\left(\left(-\lambda^{-1}, \lambda^{-2}\right)\right)$, in particular $\tilde{U}\left(\lambda^{-2}\right)=-y_{0} \tau^{-2}$. Substituting in (2.3) for $u$ the right-hand side of (2.2) yields

$$
U=\frac{1}{\tau^{2}} U \circ \frac{1}{\lambda} \circ u \circ \lambda \circ u \circ \lambda^{2}
$$

Substituting this for $U$ in (2.4) and regrouping terms gives:

$$
\tilde{U}=\frac{1}{\tau^{2}} \tilde{U} \circ \lambda \circ u \circ(-\lambda),
$$

i.e. an equation for $\tilde{U}$ identical to that satisfied by $U,(2.3)$. In particular, $U$ and $\tilde{U}$ must both vanish at any fixed point of $\lambda \circ u^{\circ}(-\lambda)$. Since $U$ is strictly decreasing in $\left(-\lambda^{-1}, \lambda^{-2}\right)$, its unique zero, namely 1 , is the only such fixed point, and

$$
\tilde{U}(1)=0 \Rightarrow \frac{1}{\lambda} u\left(\lambda^{2}\right)=1
$$

As conjugating functions of the holomorphic function $\lambda \circ u \circ(-\lambda), U$ and $\tilde{U}$ can differ only by a constant factor, which is determined by

$$
\tilde{U}^{\prime}(1)=-\frac{1}{\tau} U^{\prime}(1) \lambda u^{\prime}\left(\lambda^{2}\right)
$$

Now differentiating (2.2) at 1 gives

$$
u^{\prime}(-\lambda)=-\frac{\tau^{2}}{\lambda^{2}}
$$

and differentiating (2.2) at $-\lambda$ gives, using (2.6),

$$
u^{\prime}(-\lambda)=-u^{\prime}\left(\lambda^{2}\right)^{2} \Rightarrow u^{\prime}\left(\lambda^{2}\right)=-\frac{\tau}{\lambda} .
$$

This fixes the factor to be 1 , hence $\tilde{U}=U$ and hence, by (2.4)

$$
U=-\frac{1}{\tau} U \circ \frac{1}{\lambda} \circ u \circ \lambda^{2}
$$

In particular $t_{1}=-U\left(\lambda^{-2}\right)=y_{0} \tau^{-2}$. 


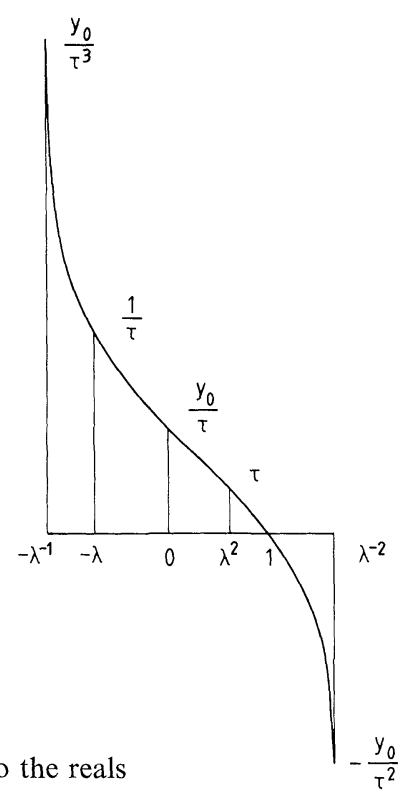

Fig. 3. Graph of $U$ restricted to the reals

This "second functional equation" gives rise to the commutativity of $\xi$ and $\eta$ in the following sense [L1]: we denote

$$
\begin{gathered}
\hat{\eta}(x)= \begin{cases}\eta(x) & \text { for } \quad x \in\left[0, x_{0} \lambda^{-2}\right] \\
G\left(-(-x)^{r}\right) & \text { for } \quad x \in\left[-x_{0} \lambda^{-1}, 0\right]\end{cases} \\
\hat{\xi}(x)=-\frac{1}{\lambda} \hat{\eta}(-\lambda x) \text { for } \quad x \in\left[-x_{0} \lambda^{-3}, x_{0} \lambda^{-1}\right],
\end{gathered}
$$

or, equivalently,

$$
\hat{u}(z)=|U(z)|^{1 / r} \operatorname{sign} U(z), \quad z \in\left[-\lambda^{-1}, \lambda^{-2}\right],
$$

and $\hat{\xi}=(-\hat{u})^{-1}$. Then for all $x \in\left[-x_{0} \lambda^{-1}, x_{0} \lambda^{-2}\right]$,

$$
\begin{aligned}
& \hat{\eta}(x)=-\frac{1}{\lambda} \hat{\eta}(\hat{\xi}(-\lambda x)), \\
& \hat{\eta}(x)=-\frac{1}{\lambda} \hat{\xi}(\hat{\eta}(-\lambda x)) .
\end{aligned}
$$

Thus $\hat{\xi}$ and $\hat{\eta}$ commute. As shown in [L1], they can be further extended by using (B).

Figure 3 summarizes the properties of $U$ implied (on $\mathbb{R}$ ) by $\mathbf{C 1 - C 3}$.

\section{The Map $M_{\lambda}$}

From now on, $\lambda \in(0,1)$ and $v \in[1,2]$ are fixed. Let

$$
\mathbf{E}_{0}(\lambda)=\left\{\psi \in-\mathbf{P}\left(\left(-\lambda^{-1}, \lambda^{-2}\right)\right): \psi(0)=1, \psi(1)=0\right\} .
$$


Let $\psi_{0} \in \mathbf{E}_{0}(\lambda)$. The function $\psi=M_{\lambda} \psi_{0}$ is, when it exists, also an element of $\mathbf{E}_{0}(\lambda)$ obtained as follows. A function $V$ is first defined by

$$
V(\zeta)=\frac{1}{\tau^{v}} \psi_{0}\left((\zeta / \alpha)^{1 / r}\right)
$$

where the constants $\tau, \alpha$, and $r$ must be determined so that

$$
V(1)=1, \quad V^{\prime}(1)=-\frac{1}{\lambda}, \quad \tau=\lambda^{r},
$$

and must satisfy

$$
r>1, \quad 1<\alpha<\frac{1}{\tau^{v}}
$$

Then $\psi$ is the solution of

$$
\psi(z)=V(\psi(-\lambda z)), \quad \psi(0)=1, \quad \psi(1)=0 .
$$

We now examine when $M_{\lambda} \psi_{0}$ can actually be defined. This discussion closely follows [E], and omits some details given there.

\section{Determination of the Constants}

Equation (3.2) and the conditions (3.3) imply that $z_{1}=\alpha^{-1 / r}$ must satisfy

$$
\psi_{0}\left(z_{1}\right)=\tau^{\nu}, \quad \frac{z_{1} \psi_{0}^{\prime}\left(z_{1}\right)}{\psi_{0}\left(z_{1}\right)}=-\frac{r}{\lambda},
$$

and since $r=(\log \tau) /(\log \lambda)$

$$
q\left(z_{1}\right)=0
$$

where $q$ is defined by

$$
q(z) \equiv \frac{\psi_{0}^{\prime}(z)}{\psi_{0}(z)}-\frac{A}{v z} \log \psi_{0}(z)
$$

Here $A \equiv 1 /(\lambda \log (1 / \lambda)) \geqq e$, and we also denote $B=\left(1-\lambda^{2}\right) A$, a decreasing function of $\lambda$ with $B(1)=2$.

We recall the "tautological bounds" which follow at once from the integral representation implied by the Herglotz property:

$$
\frac{1}{1+\lambda z} \leqq \frac{\psi_{0}(z)}{1-z} \leqq \frac{1}{1-\lambda^{2} z}
$$

valid for $0 \leqq z<\lambda^{-2}$, reversed for $-\lambda^{-1}<z \leqq 0$,

$$
\frac{\left(1-\lambda^{2}\right)}{\left(1-\lambda^{2} z\right)(1-z)} \leqq \frac{-\psi_{0}^{\prime}(z)}{\psi_{0}(z)} \leqq \frac{(1+\lambda)}{(1+\lambda z)(1-z)},
$$


valid for $-\lambda^{-1}<z<1$, reversed for $1<z<\lambda^{-2}$,

$$
-\frac{2 \lambda}{1+\lambda z} \leqq \frac{\psi_{0}^{\prime \prime}(z)}{\psi_{0}^{\prime}(z)} \leqq \frac{2 \lambda^{2}}{1-\lambda^{2} z},
$$

valid for $-\lambda^{-1}<z<\lambda^{-2}$. As $z \downarrow 0, q(z) \rightarrow \psi_{0}^{\prime}(0)(1-A / v)>0$ since $A / v \geqq e / 2$. As $z \uparrow 1$, $q(z) \sim 1 /(z-1) \rightarrow-\infty$.

This shows that $q$ has at least one zero $z_{1}$ in $(0,1)$. To show that it is unique, we compute

$$
\begin{gathered}
\frac{d}{d z} z q(z)=z \frac{\psi_{0}^{\prime}(z)}{\psi_{0}(z)} G(z) \\
G=\frac{\psi_{0}^{\prime \prime}}{\psi_{0}^{\prime}}-\frac{\psi_{0}^{\prime}}{\psi_{0}}-\frac{1}{z}\left(\frac{A}{v}-1\right) \\
G^{\prime}=\frac{\psi_{0}^{\prime \prime \prime}}{\psi_{0}^{\prime}}-\left(\frac{\psi_{0}^{\prime \prime}}{\psi_{0}^{\prime}}\right)^{2}-\frac{\psi_{0}^{\prime \prime}}{\psi_{0}}+\left(\frac{\psi_{0}^{\prime}}{\psi_{0}}\right)^{2}+\frac{1}{z^{2}}\left(\frac{A}{v}-1\right) \\
G^{\prime}=S \psi_{0}+\frac{1}{2}\left[\frac{\psi_{0}^{\prime \prime}}{\psi_{0}^{\prime}}-\frac{\psi_{0}^{\prime}}{\psi_{0}}\right]^{2}+\frac{1}{2}\left(\frac{\psi_{0}^{\prime}}{\psi_{0}}\right)^{2}+\frac{1}{z^{2}}\left(\frac{A}{v}-1\right)>0
\end{gathered}
$$

Since $G(z)$ tends to $-\infty$ when $z \downarrow 0$, and to $+\infty$ when $z \uparrow 1$, it vanishes only once in $(0,1)$. Hence $(z q(z))^{\prime}$ also vanishes only once in $(0,1)$, starts by being positive and ends up negative; $z q(z)$ increases from 0 to a unique maximum, then decreases to $-\infty$. Hence it has only one zero in $(0,1)$, denoted $z_{1}$. We define successively $\tau$, then $r$, then $\alpha$ by:

$$
\tau^{v}=\psi_{0}\left(z_{1}\right) \in(0,1), \quad r=\frac{\log \tau}{\log \lambda}>0, \quad \alpha=z_{1}^{-r}>1
$$

\section{Lower Bounds on $1 / \tau^{v}$ and $z_{1}$}

We use, as in [E], the representation

$$
\log \psi_{0}(z)=-\int \sigma(t) d t\left[\frac{1}{t-z}-\frac{1}{t}\right]
$$

where $\sigma \in L^{\infty}$ has support in $\mathbb{R}-\left(-\lambda^{-1}, 1\right)$, and $0 \leqq \sigma \leqq 1, \sigma(t)=1$ for $1<t<\lambda^{-2}$. Hence

$$
q(z)=\int \frac{\sigma(t) d t}{t-z}\left[\frac{A}{v t}-\frac{1}{t-z}\right]
$$

holds for $z \in \mathbb{C}\left(\left(-\lambda^{-1}, 1\right)\right)$. Let $z \in(0,1)$. Then the contribution to the integral in (5.2) from the negative values of $t$ is positive. For $t \geqq 1$, the integrand has the sign of

$$
\frac{A}{v}(t-z)-t
$$

This is positive, and strictly positive if $t>1$, when $z \leqq 1-v / A$. Hence

$$
z_{1}>1-v \lambda \log \frac{1}{\lambda}>\lambda^{v}
$$


The last inequality follows from the fact that $1+v \lambda \log \lambda-\lambda^{v}$ vanishes at $\lambda=1$ and its derivative in $\lambda$ is

$$
v\left(\log \lambda+1-\lambda^{v-1}\right) \leqq v(2-v) \log \lambda,
$$

and is negative in $(0,1)$.

From (5.4) we can derive crude lower bounds on $1 / \tau^{v}$ and $r$ by using (4.1), (4.3), and (4.4):

$$
\begin{gathered}
\frac{1}{\tau^{v}} \geqq \frac{1-\lambda^{2} z_{1}}{1-z_{1}} \equiv \xi_{1}, \\
\frac{1}{\tau^{v}} \geqq 1+\lambda^{2}, \\
\frac{r}{\lambda} \geqq \frac{z_{1}\left(1-\lambda^{2}\right)}{\left(1-z_{1}\right)\left(1-\lambda^{2} z_{1}\right)}, \\
r \geqq \frac{\lambda^{v+1}\left(1-\lambda^{2}\right)}{\left(1-\lambda^{v}\right)\left(1-\lambda^{2+v}\right)} \geqq \frac{\lambda^{3}}{1-\lambda^{4}} .
\end{gathered}
$$

To improve on these bounds, we observe that the integrand of (5.2) is positive if $t \in \Sigma=\mathbb{R}-\left(-\lambda^{-1}, \lambda^{-2}\right)$, since for $t \geqq \lambda^{-2}$,

$$
\frac{A}{v}(t-z)-t \geqq \lambda^{-2}\left(\frac{B}{v}-1\right)>0 .
$$

For $\tilde{\psi}_{0} \in \mathbf{E}_{0}(\lambda)$, let $\tilde{\sigma}, \tilde{q}, \tilde{z}, \tilde{\tau}, \tilde{r}$, be the objects obtained from $\tilde{\psi}_{0}$ in the same way as $\sigma, q, z_{1}, \tau, r$, from $\psi_{0}$. Suppose that $\tilde{\sigma} \leqq \sigma$. Then, for $0<z<1, \psi_{0}(z) \leqq \tilde{\psi}_{0}(z)$, and moreover, since $\tilde{\sigma}(t)=\sigma(t)=1$ on $\left(1, \lambda^{-2}\right), \tilde{q}(z) \leqq q(z)$. Thus $q\left(\tilde{z}_{1}\right) \geqq 0$, hence $\tilde{z}_{1} \leqq z_{1}$ and

$$
\tau^{\nu}=\psi_{0}\left(z_{1}\right) \leqq \psi_{0}\left(\tilde{z}_{1}\right) \leqq \tilde{\psi}_{0}\left(\tilde{z}_{1}\right)=\tilde{\tau}^{\nu}, \quad r \geqq \tilde{r} .
$$

In particular, let $\sigma_{2}$ be the characteristic function of $\left[1, \lambda^{-2}\right]$, and $\sigma_{3}$ that of $\mathbb{R}-\left(-\lambda^{-1}, 1\right)$. For $j=2,3$, define $\log \psi_{j}$ and $q_{j}$ by substituting $\sigma_{j}$ for $\sigma$ in (5.1) and (5.2), $z_{j}$ the zero of $q_{j}$ in $(0,1)$, and

$$
\tau_{j}^{\nu}=\psi_{j}\left(z_{j}\right), \quad r_{j} \equiv r_{j}(\lambda)=\log \tau_{j} / \log \lambda .
$$

Then

$$
\begin{gathered}
\psi_{2}(z)=\frac{1-z}{1-\lambda^{2} z}, \quad \psi_{3}(z)=\frac{1-z}{1+\lambda z}, \\
\tau_{3} \leqq \tau \leqq \tau_{2}, \quad r_{2}(\lambda) \leqq r \leqq r_{3}(\lambda) .
\end{gathered}
$$

To study in more detail $q_{2}$,

$$
q_{2}(z)=\frac{A}{v z} \log \left(\frac{1-\lambda^{2} z}{1-z}\right)-\frac{1-\lambda^{2}}{(1-z)\left(1-\lambda^{2} z\right)},
$$

it is convenient to use the variable $\xi=1 / \psi_{2}(z)$, i.e.

$$
\xi=\frac{1-\lambda^{2} z}{1-z}, \quad z=\frac{\xi-1}{\xi-\lambda^{2}} .
$$


This gives:

$$
\begin{gathered}
\left(1-\lambda^{2}\right) z q_{2}(z)=\chi(\xi)-\xi, \\
\chi(\xi)=\frac{B}{v} \log \xi+1+\lambda^{2}-\frac{\lambda^{2}}{\xi} .
\end{gathered}
$$

The function $\chi$ is increasing and concave on $(0, \infty)$, and $\chi(\xi)-\xi$ vanishes at $\xi=1$ and at a unique $\hat{\xi} \equiv 1 / \psi_{2}\left(z_{2}\right)>1$. In particular a sufficient condition for $r>1$ is that $r_{2}(\lambda)>1$, i.e. $\hat{\xi}>\lambda^{-v}$, i.e. $\chi\left(\lambda^{-v}\right)-\lambda^{-v}>0$. This gives

$$
\lambda^{v-1}\left(1-\lambda^{2}\right)-\left(1-\lambda^{v}\right)\left(1-\lambda^{2+v}\right) \equiv\left(1-\lambda^{v}\right)\left(\lambda^{2+v}+\lambda^{v-1}-1\right)+\left(\lambda^{v}-\lambda^{2}\right) \lambda^{v-1}>0 \text {. }
$$

This condition is satisfied for all $\lambda \in(0,1)$, when $v=1$, but for $v>1$, it is only satisfied for $\lambda>\lambda_{0}(v)>0$, where $\lambda_{0}(v)$ is the unique zero $<1$ of the function of $\lambda$ in (5.17). This function is plotted in Fig. 1 (upper curve). In particular, for $v=2$, the condition (5.17) is equivalent to

$$
\lambda+\lambda^{4}-1>0
$$

i.e. $\lambda>\lambda_{0}(2) \sim 0.72449 \ldots$.

On the other hand we expect (see in particular [JR]) solutions of the functional equations to exist as soon as

$$
\lambda^{v}+\lambda^{v-1}-1>0
$$

This is obtained by trying to find a trivial solution with $r=1$, i.e. to set:

$$
\psi(z)=1-z, \quad V(\zeta)=\frac{1}{\lambda^{v}}(1-\zeta / \alpha)
$$

Then $V(1)=1, V^{\prime}(1)=-1 / \lambda$ require

$$
\frac{1}{\alpha}=1-\lambda^{v}=\lambda^{v-1}
$$

If $\lambda^{v}+\lambda^{v-1}-1=0$, the functions appearing in (5.20) satisfy (3.5). The discrepancy between (5.19) and (5.17), which cannot be improved in $\mathbf{E}_{0}(\lambda)$, shows that a narrower domain must be sought for $M_{\lambda}$. Here, however, we shall only consider, from now on, values $\lambda>\lambda_{0}(v)$. In this case there is a lower bound $r_{2}(\lambda) \equiv r_{2}(\lambda, v)>1$ for $r$. Moreover the crude bound (5.8) shows that $r_{2}(\lambda, v) \rightarrow+\infty$ as $\lambda \rightarrow 1$.

Lower Bound on $\alpha$. We note that, as a consequence of (4.6) and (5.4),

$$
1<\alpha<\frac{1}{\tau^{v}}
$$

A lower bound for $\alpha$, strictly greater than 1 , and uniform as $\lambda \rightarrow 1$ can be obtained from (5.7) and (5.4):

$$
\frac{z_{1}}{1-z_{1}} \leqq \frac{r\left(1-\lambda^{2+v}\right)}{\lambda\left(1-\lambda^{2}\right)} \leqq r\left(\lambda+\frac{1}{\lambda}\right)
$$


hence, denoting temporarily $m=\lambda+1 / \lambda$,

$$
z_{1} \leqq 1-\frac{1}{r m+1} \leqq \exp \left(-\frac{1}{r m+1}\right)
$$

and

$$
\alpha=z_{1}^{-r} \geqq \exp \left(\frac{1}{\lambda+1 / \lambda+1 / r}\right) .
$$

For $r>1$ we also get

$$
\frac{1}{z_{1}^{r}} \geqq\left(1+\frac{1}{r m}\right)^{r} \geqq 1+\frac{1}{m},
$$

hence

$$
\alpha \geqq 1+\frac{\lambda}{1+\lambda^{2}} .
$$

\section{Definition of $V$ and $W$}

We can now define $V$ by (3.2) and, since $r>1,-V$ is a Herglotz function. We define $W=V \circ V$, and $\hat{W}(\zeta)=1-W(1-\zeta)$. Both $W$ and $\hat{W}$ are Herglotz functions and

$$
\begin{gathered}
V \in-\mathbf{P}\left(\left(0, \alpha \tau^{-2}\right)\right), \quad W \in \mathbf{P}((0, \alpha)), \\
V(1)=1, \quad V^{\prime}(1)=-\frac{1}{\lambda}, \quad V(0)=\frac{1}{\tau^{v}}, \quad V(\alpha)=0, \\
W(1)=1, \quad W^{\prime}(1)=\frac{1}{\lambda^{2}}=\hat{W}^{\prime}(0), \quad \hat{W}(0)=0, \\
W(\alpha)=\frac{1}{\tau^{v}}, \quad W(0)=V\left(\frac{1}{\tau^{v}}\right) .
\end{gathered}
$$

Note that $1 / \tau^{v}$ is in the real interval of analyticity $\left(0, \alpha \tau^{-2}\right)$ of $V$ because of (5.21), and, for the same reason, $V\left(1 / \tau^{v}\right)<0$, i.e. $\hat{W}(1)>1$. A better lower bound for $\hat{W}(1)$ is needed, and will be derived in the next section.

\section{Lower Bound for $\hat{W}(\zeta)$ in $(0,1)$}

We use the following identities, where we denote $z=(\zeta / \alpha)^{1 / r}$ :

$$
\begin{gathered}
V^{\prime}(\zeta)=\frac{1}{\tau^{v} r} \frac{z}{\zeta} \psi_{0}^{\prime}(z), \\
-\zeta r \frac{V^{\prime \prime}(\zeta)}{V^{\prime}(\zeta)}=r-1-z \frac{\psi_{0}^{\prime \prime}(z)}{\psi_{0}^{\prime}(z)}, \\
S V(\zeta)=S \psi_{0}(z)\left(\frac{z}{r \zeta}\right)^{2}+\frac{1}{2 \zeta^{2}}\left(1-r^{-2}\right) .
\end{gathered}
$$

Inserting into (7.2) the first inequality in (4.5) gives

$$
-\zeta \frac{V^{\prime \prime}(\zeta)}{V^{\prime}(\zeta)} \leqq 1 \text { for } 0<\zeta<\alpha \tau^{-1},
$$


in particular

$$
-\frac{V^{\prime \prime}(1)}{V^{\prime}(1)} \leqq 1
$$

and hence

$$
\frac{\hat{W}^{\prime \prime}(0)}{\hat{W}^{\prime}(0)}=\left(\frac{1}{\lambda}-1\right) \frac{V^{\prime \prime}(1)}{V^{\prime}(1)} \geqq-\left(\frac{1}{\lambda}-1\right) .
$$

For $0<\zeta<\alpha \tau^{-2},(7.3)$ gives

$$
S V(\zeta) \geqq \frac{1-r^{-2}}{2 \zeta^{2}}
$$

hence, for $1-\alpha<\zeta<1$,

$$
S \hat{W}(\zeta)=S W(1-\zeta) \geqq S V(1-\zeta) \geqq \frac{1-r^{-2}}{2(1-\zeta)^{2}} .
$$

This implies, for $\zeta \in[0,1)$,

$$
\frac{d}{d \zeta} \frac{\hat{W}^{\prime \prime}(\zeta)}{\hat{W}^{\prime}(\zeta)} \geqq \frac{1}{2}\left(1-r^{-2}\right),
$$

and, using (7.6),

$$
\begin{gathered}
\frac{\hat{W}^{\prime \prime}(\zeta)}{\hat{W}^{\prime}(\zeta)} \geqq-\left(\frac{1}{\lambda}-1\right)+\frac{1}{2}\left(1-r^{-2}\right) \zeta, \\
\log \hat{W}^{\prime}(\zeta) \geqq\left[-2 \log \lambda-\left(\lambda^{-1}-1\right) \zeta\right]+\frac{1}{4}\left(1-r^{-2}\right) \zeta^{2} .
\end{gathered}
$$

For $0 \leqq \zeta<1$ and $\lambda>1 / e$, the bracket in (7.9) is positive, so that

$$
\hat{W}(\zeta) \geqq \zeta\left(1+a \zeta^{2}\right), \quad a=\frac{1}{12}\left(1-r^{-2}\right) .
$$

On the other hand, using $\hat{W} \in \mathbf{P}((1-\alpha, 1))$ and $\hat{W}(0)=0, \hat{W}^{\prime}(0)=\lambda^{-2}$, we find, for $0 \leqq \zeta<1$,

$$
\hat{W}(\zeta) \geqq \frac{\zeta(\alpha-1)}{\lambda^{2}(\alpha-1+\zeta)} \geqq \frac{\zeta}{\lambda\left(1+\lambda+\lambda^{2}\right)},
$$

where the last inequality uses the bound (5.24). This easily implies that (7.10) also holds for $\lambda \leqq 1 / e$, i.e. for all $\lambda>\lambda_{0}(v)$.

\section{Definition of $\psi$}

The unique function $\psi$ satisfying

$$
\psi \in \mathbf{E}_{0}(\lambda), \quad \psi(z)=V(\psi(-\lambda z)),
$$

can now be constructed by the same arguments as in [E]. 


\section{Definition of $\boldsymbol{H}$ and $\boldsymbol{H}_{0}$}

We now define the functions

$$
\begin{gathered}
H_{0}(w) \equiv 1-\hat{H}_{0}(w)=\psi_{0}\left(e^{\beta w}\right), \quad \beta=\log \frac{1}{\lambda}, \\
H(w) \equiv 1-\hat{H}(w)=\psi\left(e^{\beta w}\right) .
\end{gathered}
$$

Each of these functions is holomorphic in $\mathbb{C}$ minus the cuts

$$
\left\{w: e^{\beta w}-\lambda^{-2} \in \mathbb{R}_{+}\right\} \cup\left\{w: e^{\beta w}+\lambda^{-1} \in \mathbb{R}_{-}\right\},
$$

and in particular in the domain

$$
\left\{w \in \mathbb{C}:|\operatorname{Im} w|<\pi / \log \frac{1}{\lambda} \text { and } w \notin 2+\mathbb{R}_{+}\right\} .
$$

In this subdomain, $\operatorname{Im} w>0 \Rightarrow \operatorname{Im} H(w)<0$, and similarly for $H_{0}$. For the same reasons as in [E], $H$ and $H_{0}$ are decreasing on $(-\infty, 2)$, vanish at 0 , tend to 1 at $-\infty$, and are concave on $(-\infty, 1)$. These functions satisfy

$$
\begin{array}{cl}
H(w)=W(H(w-2)), & \hat{H}(w)=\hat{W}(\hat{H}(w-2)), \\
H(0)=H_{0}(0)=0, & \hat{H}(0)=\hat{H}_{0}(0)=1,
\end{array}
$$

and

$$
V(\zeta)=\frac{1}{\tau^{v}} H_{0}\left(\frac{\log (\zeta / \alpha)}{\log (1 / \tau)}\right)
$$

We denote

$$
\zeta_{1}=-w_{1}=\frac{\log \alpha}{\log (1 / \tau)}=\frac{\log z_{1}}{\log \lambda} .
$$

Then

$$
H_{0}\left(-\zeta_{1}\right)=\tau^{v}, \quad \frac{H_{0}^{\prime}\left(-\zeta_{1}\right)}{H_{0}\left(-\zeta_{1}\right)}=-\frac{1}{\lambda} \log \frac{1}{\tau},
$$

and the concavity of $H_{0}$ implies

$$
\frac{1}{\lambda} \log \frac{1}{\tau} \leqq \frac{1}{\zeta_{1}},
$$

i.e., by $(9.6)$,

$$
\log \alpha \leqq \lambda, \quad \alpha \leqq e^{\lambda} .
$$

The same arguments as in $[\mathrm{E}]$ show that, for all $w \in \mathbb{R}$,

$$
\begin{gathered}
\hat{H}^{\prime}(w) \geqq \frac{a^{\prime}}{2} \hat{H}(w)^{3}, \\
\hat{H}(w) \leqq\left(1-a^{\prime} w\right)^{-1 / 2},
\end{gathered}
$$


where

$$
a^{\prime}=\frac{a}{1+3 a}, \quad a=\left[1-r_{2}(\lambda)^{-2}\right] / 12
$$

\section{Lower Bound on $\tau$}

This follows the same line as in $[\mathrm{E}]$ : we denote

$$
k=\int_{\Sigma} \frac{\sigma(t) d t}{t(t-1)}=\log \left(\frac{-1}{\psi_{0}^{\prime}(1)\left(1-\lambda^{2}\right)}\right),
$$

and note that letting $z$ tend to 1 in the inequalities (4.3) gives $(1+\lambda)^{-1} \leqq-\psi_{0}^{\prime}(1)$, hence

$$
k \leqq \log \frac{1}{1-\lambda} \leqq \frac{\lambda}{1-\lambda} .
$$

Denoting $q=q_{1}+q_{2}$, [see (5.3)] we write

$$
q_{1}(z)=\int_{\Sigma} \frac{\sigma(t) d t}{t(t-1)} I(t, z)
$$

with

$$
I(t, z)=\frac{t-1}{t-z}\left[\frac{A}{v}-\frac{t}{t-z}\right] .
$$

It has already been noted that, for $0<z<1$, and $t \in \Sigma$, the bracket in (10.4) is positive. Moreover

$$
\frac{\partial}{\partial t} I(t, z)=\frac{1-z}{(t-z)^{2}}\left[\frac{A}{v}-\frac{t}{t-z}\right]+\frac{t-1}{t-z} \frac{z}{(t-z)^{2}} \geqq 0 .
$$

For $t \rightarrow \pm \infty, I(t, z) \rightarrow A / v-1$. Hence, for $t \in \Sigma, I(t, z)$ reaches its maximum at $t=-1 / \lambda$, so that

$$
I(t, z) \leqq \frac{1+\lambda}{1+\lambda z}\left[\frac{A}{v}-\frac{1}{1+\lambda z}\right] \leqq \frac{A}{v}(1+\lambda),
$$

and

$$
q_{1}(z) \leqq k \frac{A}{v}(1+\lambda), \quad 0 \leqq z \leqq 1
$$

It follows that, with $\xi$ as in (5.14),

$$
\left(1-\lambda^{2}\right) z q(z) \leqq \chi(\xi)-\xi+\frac{B}{v}(1+\lambda) k .
$$

In view of (10.2), it follows that there exists an upper bound $\xi_{\max }$ for $\xi_{1}$ which depends only on $\lambda$. (Later, by imposing further restrictions on $\psi_{0}$, we shall obtain a $\xi_{\max }$ bounded as $\lambda \rightarrow 1$.) The bound

$$
\tau^{\nu}=\psi_{0}\left(z_{1}\right) \geqq \frac{1-z_{1}}{1+\lambda z_{1}}=\frac{1-\lambda}{\xi_{1}-\lambda}
$$


gives

$$
\frac{1}{\tau^{v}} \leqq \frac{\xi_{\max }-\lambda}{1-\lambda}
$$

i.e. an upper bound for $\tau^{-v}$ depending only on $\lambda$.

\section{Upper Bound on $\hat{W}(1)$}

Recall

$$
\begin{gathered}
W(0)=V\left(\frac{1}{\tau^{v}}\right)=\frac{1}{\tau^{v}} \psi_{0}(p), \\
1<p=\left(\tau^{v} \alpha\right)^{-1 / r}=z_{1} \lambda^{-v}<\lambda^{-2} .
\end{gathered}
$$

Using the bound (4.3) on $\psi_{0}$, we find:

$$
-\psi_{0}(p) \leqq \frac{1}{\lambda^{v}} \frac{z_{1}-\lambda^{v}}{1-\lambda^{2-v} z_{1}} \leqq \frac{1}{\lambda^{v}} \frac{z_{1}-\lambda^{v}}{1-z_{1}} \leqq \frac{1}{\lambda^{2}} \frac{z_{1}-\lambda^{2}}{1-z_{1}}
$$

Recall that

$$
z_{1} \leqq \frac{\xi_{\max }-1}{\xi_{\max }-\lambda^{2}}
$$

hence

$$
\begin{gathered}
-\psi_{0}(p) \leqq \frac{1}{\lambda^{2}}\left(\xi_{\max }-1-\lambda^{2}\right), \\
\hat{W}(1) \leqq 1+\frac{1}{\lambda^{2} \tau^{\nu}}\left(\xi_{\max }-1-\lambda^{2}\right) .
\end{gathered}
$$

\section{Uniform Lower Bounds on $\tau$ as $\lambda \rightarrow 1$}

The preceding sections have shown that: for all $\lambda>\lambda_{0}(v)$, there exists a $C_{1}(\lambda)<\infty$ such that

$$
M_{\lambda}\left(\mathbf{E}_{0}(\lambda)\right) \subset \mathbf{E}_{1}(\lambda)
$$

where $\mathbf{E}_{1}(\lambda)$ is the compact convex subset of $\mathbf{E}_{0}(\lambda)$ defined by

$$
\begin{aligned}
\mathbf{E}_{1}(\lambda)=\{ & \left\{\psi_{0} \in \mathbf{E}_{0}(\lambda):\left|\psi_{0}(z)\right| \leqq C_{1}(\lambda) \text { for all } z \in\left(-\lambda^{-1}, \lambda^{-2}\right),\right. \\
& \left.(2 z \log \lambda) \psi_{0}^{\prime}(z) \geqq a^{\prime}\left[1-\psi_{0}(z)\right]^{3} \text { for } z \in[0,1]\right\} .
\end{aligned}
$$

Here $a^{\prime}$ is the quantity defined in (9.11). It depends on $\lambda$ but tends to $1 / 15$ as $\lambda \rightarrow 1$.

If we now assume that $\psi_{0} \in \mathbf{E}_{1}(\lambda)$, then (9.9) and (9.11) hold with $\hat{H}$ and $\hat{H}^{\prime}$ replaced by $\hat{H}_{0}$ and $\hat{H}_{0}^{\prime}$, so that

$$
-\psi_{0}^{\prime}(1) \geqq \frac{a^{\prime}}{2 \log (1 / \lambda)}, \quad k \leqq \log \left(\frac{2 \log (1 / \lambda)}{a^{\prime}\left(1-\lambda^{2}\right)}\right),
$$


where $k$ is as in (10.1). Together with (10.2), this shows that there is a constant $K$, independent of $\lambda$, such that (for $\lambda>\lambda_{0}(v)$ )

$$
\left(1-\lambda^{2}\right) z q(z) \leqq \frac{B}{v} \log \xi+K-\xi
$$

Hence there is a $\xi_{\max }$ independent of $\lambda>\lambda_{0}(v)$ such that $\xi_{1} \leqq \xi_{\max }$. The bound (10.10) holds, of course, with this new $\xi_{\max }$, but is worthless when $\lambda \rightarrow 1$. With $\zeta_{1}$ as in (9.6), we find

$$
\begin{gathered}
\zeta_{1}=\frac{1}{\log \lambda} \log \left(1-\frac{1-\lambda^{2}}{\xi_{1}-\lambda^{2}}\right), \\
\zeta_{1} \geqq \frac{\left(1-\lambda^{2}\right)}{\left(\xi_{\max }-\lambda^{2}\right) \log (1 / \lambda)} \geqq \frac{\lambda(1+\lambda)}{\xi_{\max }-\lambda^{2}} .
\end{gathered}
$$

Moreover

$$
\tau^{\nu}=H_{0}\left(-\zeta_{1}\right) \geqq 1-\left(1+a^{\prime} \zeta_{1}\right)^{-1 / 2}
$$

hence

$$
\frac{1}{\tau^{\nu}} \leqq \frac{3}{2}+\frac{2}{a^{\prime} \zeta_{1}} \leqq \frac{3}{2}+\frac{2\left(\xi_{\max }-\lambda^{2}\right)}{a^{\prime} \lambda(1+\lambda)}
$$

Combining this with the results of the preceding sections, we obtain

Lemma. There exists a continuous function $\lambda \rightarrow C(\lambda)$ on $\left(\lambda_{0}(v), 1\right]$ such that, for all $\lambda \in\left(\lambda_{0}(v), 1\right)$,

$$
M_{\lambda}\left(\mathbf{E}_{1}(\lambda)\right) \in \mathbf{E}(\lambda),
$$

where $\mathbf{E}(\lambda)$ is the compact convex subset of $\mathbf{E}_{1}(\lambda)$ defined by

$$
\begin{aligned}
\mathbf{E}(\lambda)=\{ & \left\{\psi_{0} \in \mathbf{E}_{0}(\lambda):\left|\psi_{0}(z)\right| \leqq C(\lambda) \text { for all } \mathrm{z} \in\left(-\lambda^{-1}, \lambda^{-2}\right),\right. \\
& \left.(2 z \log \lambda) \psi_{0}^{\prime}(z) \geqq a^{\prime}\left[1-\psi_{0}(z)\right]^{3} \text { for } z \in[0,1]\right\} .
\end{aligned}
$$

\section{Existence of Fixed Points}

The continuity of $M_{\lambda}$ is verified exactly as in [E], and the Schauder-Tikhonov theorem proves the existence of fixed points of $M_{\lambda}$ in $\mathbf{E}(\lambda)$ for all $\lambda>\lambda_{0}(v)$, and thus of solutions of (1.1-1.3) satisfying C1-C3. The same arguments as in [E] show the existence of the Eckmann-Wittwer functions for $\lambda=1$ for all $v$, in particular for the case of circle maps. The solutions for $\lambda<1$ have analyticity properties which can be studied by the method of [EL]. The above results do not include a proof of the existence of solutions for the case of main interest, i.e. $v=2$ and $r=3$, although the value of $\lambda$ obtained in the proofs of Lanford and de la Llave [LL], and Mestel [M], namely $0.7760513 \ldots$ is among the values for which our method works. If we admit, for this reason or for the reason given in Sect. 1, in view of the hyperbolicity results of Mestel, that the solution of [LL] and [M] satisfies $\mathbf{C 1 - C 3}$, as well as the injectivity of $\psi$, then the method of [EL] can be applied in a straightforward way to study its analyticity properties. 
Acknowledgements. We wish to thank O. Lanford for many instructive discussions. We also wish to thank the IHES and the University of Geneva for their kind hospitality in the course of this work. The support of the Fonds National Suisse is gratefully acknowledged.

\section{References}

[ACT] Arnéodo, A., Coullet, P., Tresser, Ch.: A possible new mechanism for the onset of turbulence. Phys. Lett. 81 A, 197 (1981)

[E] Epstein, H.: New proofs of the existence of the Feigenbaum functions. Commun. Math. Phys. (to appear)

[EL] Epstein, H., Lascoux, J.: Analyticity properties of the Feigenbaum function. Commun. Math. Phys. 81, 437-453 (1981)

[FKS] Feigenbaum, M.J., Kadanoff, L.P., Shenker, S.J.: Quasi-periodicity in dissipative systems. A renormalisation group analysis. Physica 5 D, 370-386 (1982)

[JR] Jonker, L., Rand, D.: Universal properties of maps of the circle with $\varepsilon$-singularities. Commun. Math. Phys. 90, 273-292 (1983)

[L1] Lanford, O.E. III: Functional equations for circle homeomorphisms with golden ratio rotation number. J. Stat. Phys. 34, 57-73 (1984)

[L2] Lanford, O.E. III: Renormalisation group methods for circle mappings. Proceedings of the conference on statistical mechanics and field theory: mathematical aspects. Groningen 1985. Lecture Notes in Physics. Berlin, Heidelberg, New York: Springer (to appear)

[LL] Lanford, O.E. III, de la Llave, R.: In preparation

[M] Mestel, B.: Ph.D. Dissertation, department of mathematics. Warwick University (1985)

[N] Nauenberg, M.: On fixed points for circle maps. Phys. Lett. AB 92, 319-320 (1982)

[ORSS] Ostlund, S., Rand, D., Sethna, J., Siggia, E.: Universal properties of the transition from quasi-periodicity to chaos in dissipative systems. Physica 8D, 303-342 (1983)

Communicated by A. Jaffe

Received June 2, 1986 
